\title{
Layered Double Hydroxides as nano additives in poly(e-caprolactone)
}

Arao Manhique $^{1,3}$, Walter W Focke ${ }^{2}$, and Carvalho Madivate ${ }^{3}$ Institute of Applied Materials, Department of Chemistry ${ }^{1}$ and Department of Chemical Engineering ${ }^{2}$, University of Pretoria, Pretoria - South Africa

${ }^{3}$ Department of Chemistry, Universidade Eduardo Mondlane, Maputo - Mozambique

\begin{abstract}
Poly( $\varepsilon$-caprolactone) MgAl - layered double hydroxides (MgAl-LDH) nanocomposites were prepared by melt intercalation. Two organically modified MgAl-LDH, stearic acid (stearate) and sodium dodecyl sulphate (SDS), were used. Nanocomposites morphology was studied by XRD and TEM. Thermal properties were analysed by TG and DSC. Although both systems showed presence of a mixed morphology, exfoliated and intercalated, MgAl-LDH stearate nanocomposites showed a better dispersion of inorganic platelets. TEM images showed the presence of individual platelets in the PCL matrix. MgAl-LDH SDS nanocomposites
\end{abstract}

\section{Introduction}

Poly( $\varepsilon$-caprolactone) (PCL) is a biodegradable polymer with potential applications in the field of bioengineering and packaging. It features a melting range of $59-64{ }^{\circ} \mathrm{C}$ and a low glass transition temperature $\left(\approx-60^{\circ} \mathrm{C}\right)$. The low melting point complicates processing and its poor mechanical properties have hampered widespread use (Jimenez et al 1997; Penha et al 2006; Woodruff and Hutmacher, 2010). The addition of small amounts of benign inorganic fillers is may lead to an improvement of some of these characteristics (Jimenez et al 1997). Layered double hydroxides (LDH) have been used in this regard.

Property improvement by LDH or by other nanofillers is intrinsically dependent on the compatibility of the filler with parent polymer. The compatibility will impact directly in terms of the degree of exfoliation that can be achieved and therefore it will influence the property improvement. A better compatibility can be achieved by intercalation of suitable organic molecules into the clay layers.

Organoclay dispersion is generally achieved in three different ways: (1) direct intercalation, where the polymer is directly intercalated in the clay matrix; (2) in situ polymerization, 
where monomers are imbibed into clay structure prior to polymerization; (3) and coprecipitation, which involves simultaneous formation of clay layers and intercalation (Pinnavaia and Beall, 2001).

Nanocomposites are generally characterized by XRD and TEM. A complete exfoliation may result in the disappearance of the basal reflections. It is important to note, however, that other changes can result in basal reflections disappearing. TEM provides direct information on the morphology of the nanocomposites but the representativeness of the sample can be questioned (Wagener and Reisinger, 2003).

Direct intercalation by melt processing is the most environmentally and commercially advantageous processing technique for nanocomposites (Vaia et al, 1993; Vaia and Gianelis, 1997). However Nhlapo et al (2007) reported a phase transition above $85^{\circ} \mathrm{C}$ and complete melting above $120^{\circ} \mathrm{C}$ in $\mathrm{LDH}$-stearate. This makes the material unfit for melt processing with conventional thermoplastics that require higher processing temperatures. This investigation was aimed to study the intercalation of LDH-stearate in the low melting poly( $\varepsilon$-caprolactone) polymer. The hope of this investigation was a clearer picture of propensity of LDH-stearate to exfoliation.

\section{Experimental}

\subsection{Materials}

Poly(E-caprolactone) (PCL 6500), with 50000 average molecular mass, was supplied by Solvay Interox Ltd (UK). Two nanofillers were used. Layered double hydroxide modified with stearic acid (LDH-Stearate) and with sodium dodecyl sulphate (LDH-SDS). The LDH is a commercial product from Chamotte Holdings South Africa with composition corresponding to $1,21: 1$ (Mg:Al) in mol basis. Modification was conducted as reported by Nhlapo et al. (2007).

\subsection{Compounding and Injection Moulding}

The organoclay and polymer were dried overnight in a vacuum oven at $60^{\circ} \mathrm{C}$. The dried mixture was compounded in a Brabender Plasticorder (Duisburg EHT 50, German) for 6 min 
at $120 \mathrm{rpm}$ speed. Compounded samples were injection moulded in a DACA Microinjector (USA) at $90^{\circ} \mathrm{C}$.

\subsection{Characterization}

X-ray diffraction analyses were conducted in a XRD $3003 \theta / \theta$ (Seifert-FPM Freiberg/Sa.) instrument, over the range of $2 \theta=0.5$ to $30^{\circ}$ in symmetric reflection, long secondary Soller collimator. $\mathrm{Cu}-\mathrm{K} \alpha$ radiation (monochromatization by a primary multilayer system) was used at $40 \mathrm{kV}$ and $30 \mathrm{~mA}$. The step-scan mode was $10 \mathrm{~s}$ per point.

For scanning electron microscopy a Leo 435 VP instrument from Carl Zeiss SMT was used. The sample was dispersed on a carbon tape in a sample holder. The sample was coated with approximately $15 \mathrm{~nm}$ platinum using a sputtercoater (SCD 500 from Baltec). Optical microscopy pictures were taken in an Olympus (type $\mathrm{BH}-2$ ) equipped with camera Olympus DP71 software analysis. The hot stage used was a Mettler FP82T. A heating rate of $10^{\circ} \mathrm{C} / \mathrm{min}$ was applied. Rheological tests were conducted in a Gottfert Rheograph 2002 at 90 ㅇ. A $30 \mathrm{~mm} \times 1 \mathrm{~mm}$ capillary was used. For dynamic mechanical analysis an EPLEXOR 150N (GABO, German) was used. The temperature range used -80 to $50{ }^{\circ} \mathrm{C}$, soak time $300 \mathrm{~s}$, heating rate of $3^{\circ} \mathrm{C} / \mathrm{min}$ at a frequency of $10 \mathrm{~Hz}$, in tension force mode.

\section{Results and Discussion}

\subsection{Characterization of the Composites}

\subsubsection{X-Ray Powder Diffraction and TEM}

XRD was used to verify the exfoliation of the LDH in the composites. A completely delaminated or exfoliated nanocomposite would result in the complete loss of the XRD reflections. However not only exfoliation can result in XRD reflections collapse, low concentrations and loss of symmetry can lead to such a collapse. Difractograms of the PCLLDH stearate and the PCL-LDH SDS composites are presented in Fig. 1. No significant changes were observed in position of the XRD peaks. However higher order peaks became weaker or even disappeared. This indicated that exfoliation was not completely achieved and that LDH crystalline structure was preserved. Based on these XRD results one can assume at best that 
a microcomposite structure was achieved. A clearer understanding of the morphology can be obtained by analysing TEM micrographs.
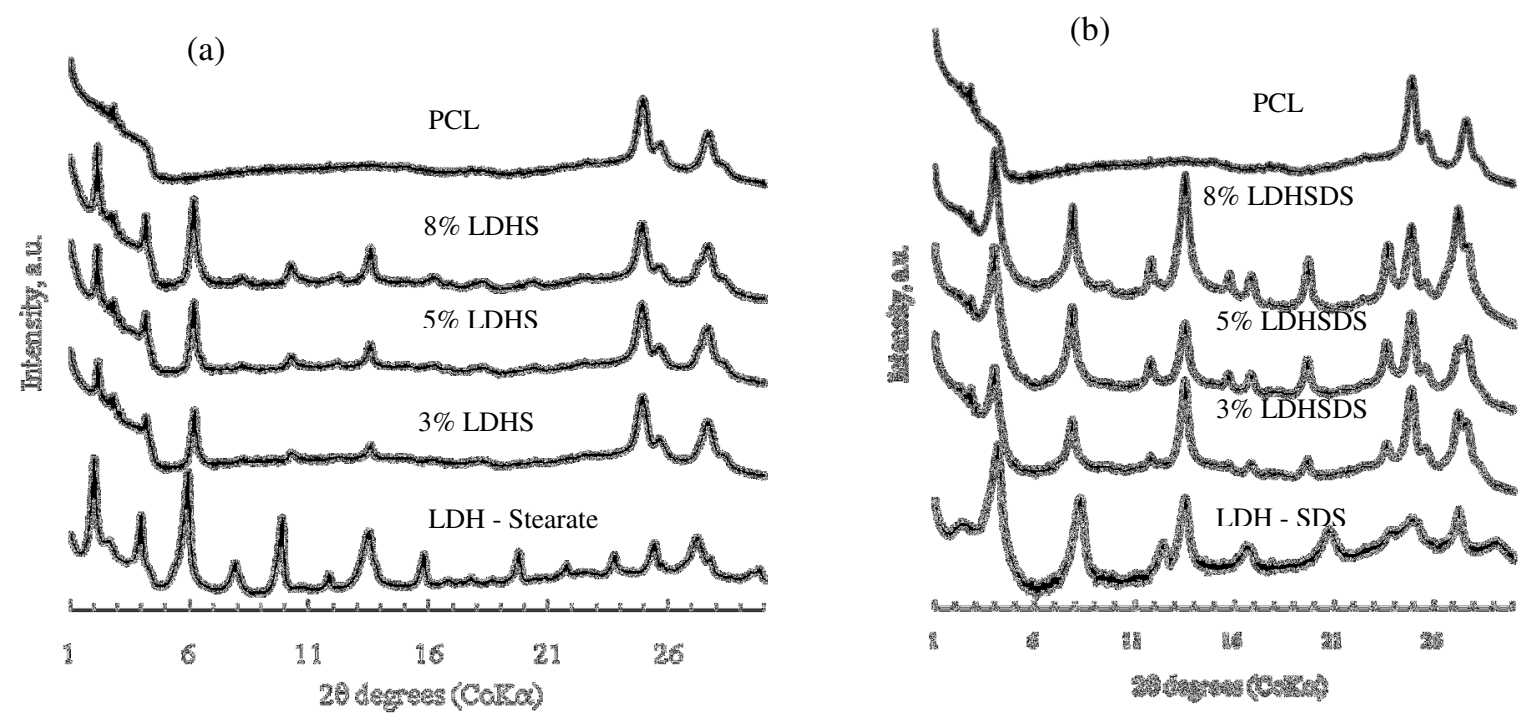

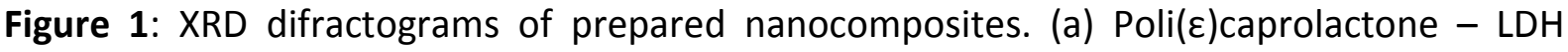
stearate; (b) Poli( $\varepsilon$ )caprolactone - LDHSDS.

A panoramic view (Fig. 2) of the composites showed a better and much uniform distribution of filler in LDH - SDS composites, Fig. 2a, compared to LDH stearate composites. LDH SDS composites, according to this image seem to have a good distribution of LDH particles in PCL matrix as opposite to LDH stearate composites. However this distribution can also indicate a poorer distribution at the nanometer scale. Thus in LDH stearate a better nanoscale distribution may have been achieved.
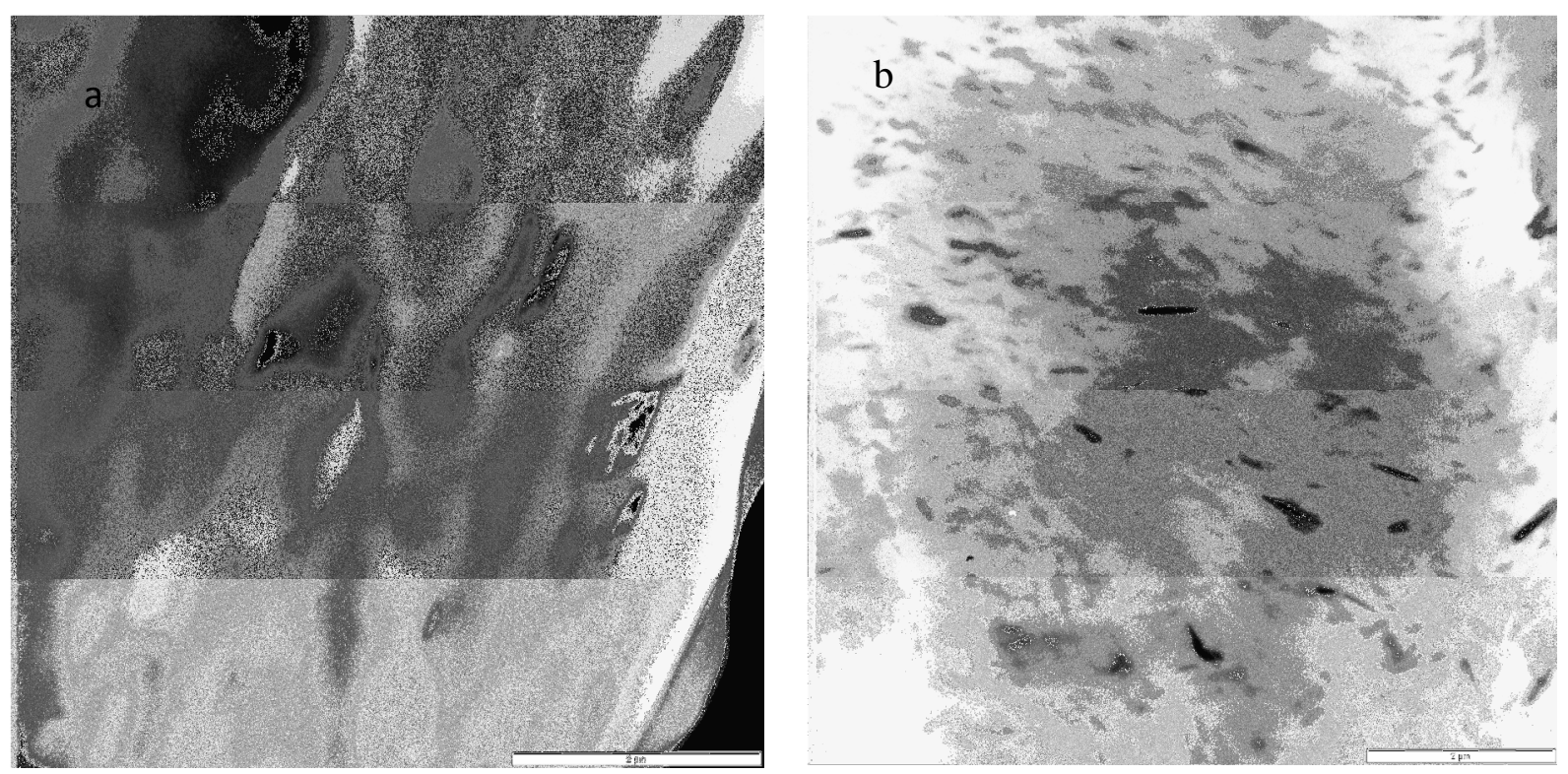
Figure 2: Low magnification TEM image of PCL composites prepared in this work. (a) LDH stearate; (b) LDH SDS.

In fact medium magnification TEM images showed the presence of a higher density of tactoids in LDH SDS composites compared to LDH stearate (Fig. 3). However individually dispersed particles (highlighted) are present in polymer matrix of both nanocomposites. Here one can see that a mixed morphology was achieved. The LDH platelets are present in the form of individual particles as well as tactoids.
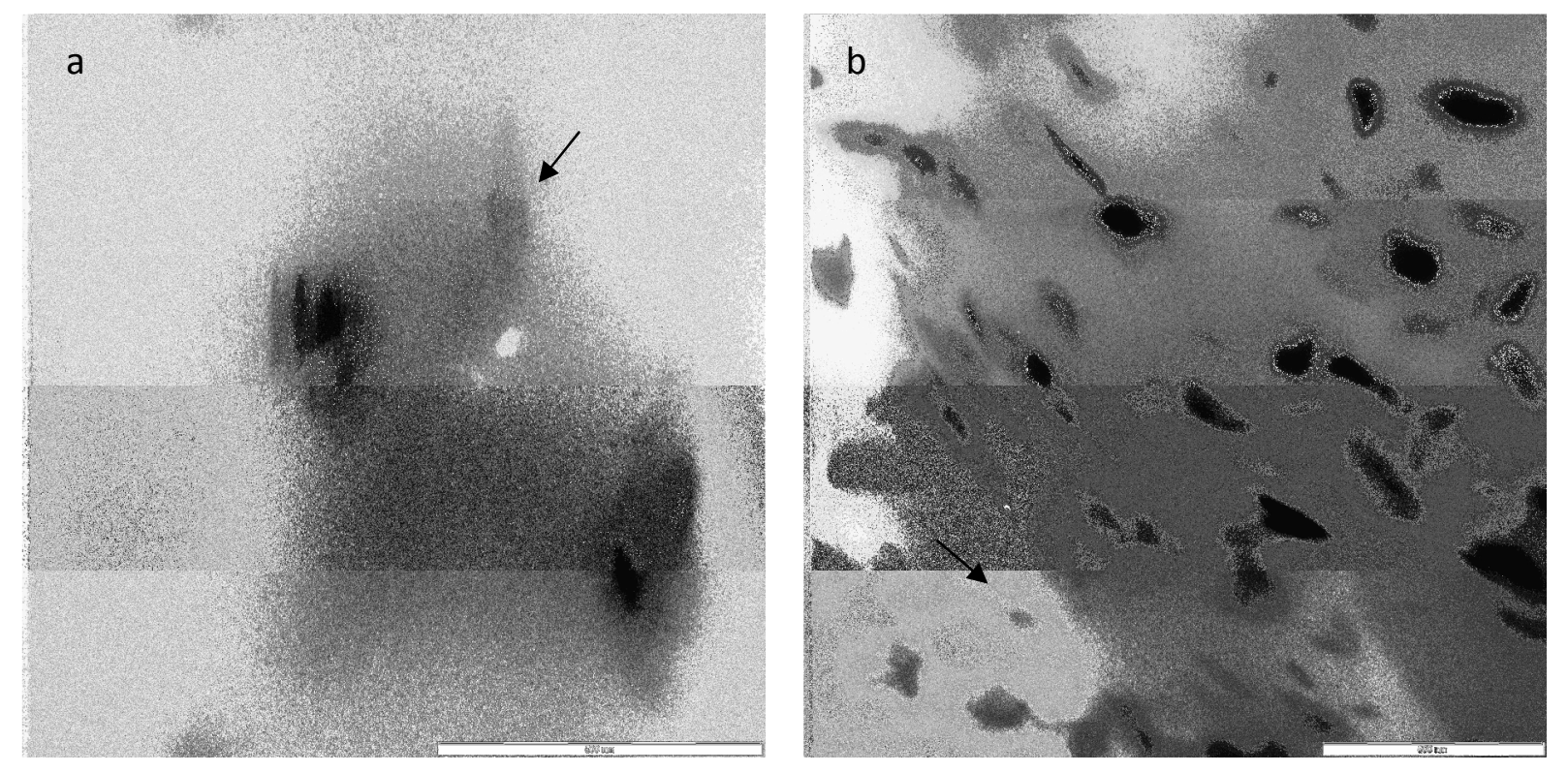

Figure 3: TEM images of poly( $(\varepsilon)$ caprolactone LDH stearate (a), LDH SDS (b) nanocomposites, $500 \mathrm{~nm}$ magnification.

A higher magnification image shows that some LDH stearate crystals in nanocomposites are present as few groups of crystals are assembled as piles with different arrangements (Fig. 4a). LDH platelets in LDH SDS nanocomposites are present as tactoids, indicating a lesser extent of exfoliation/intercalation in this system. These tactoids are sparsely dispersed in the polymer matrix (Fig. 4b). These particles will show a limited symmetry resulting in the disappearance of higher order reflections on the XRD diffractogram as was observed by Costa et al. (2005). 

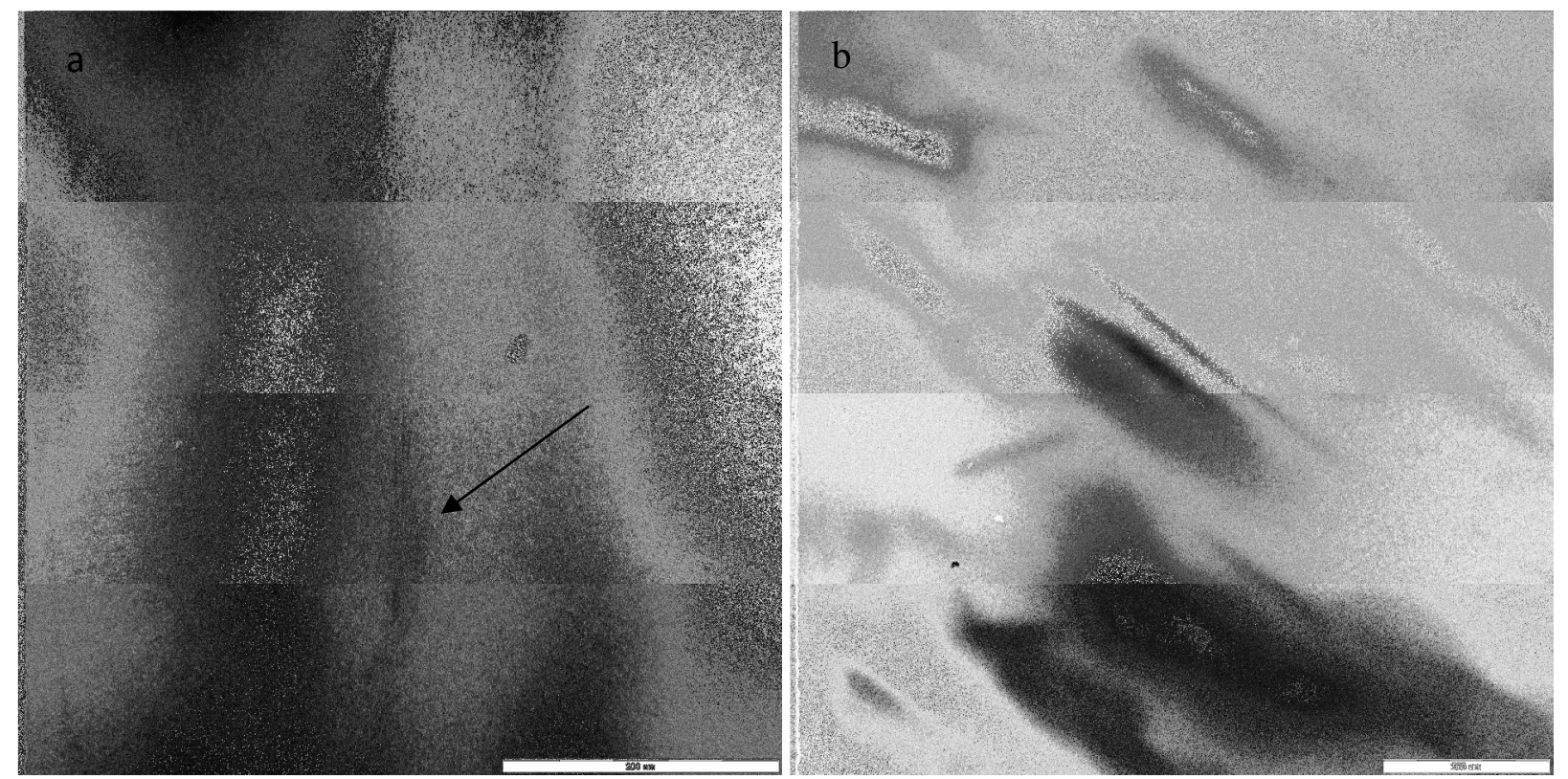

Figure 4: High magnification TEM images of poly( $(\varepsilon)$ caprolactone LDH stearate (a), LDH SDS (b), $200 \mathrm{~nm}$ magnification.

\subsection{FTIR Analysis}

FTIR spectra are shown in Fig. 5. The bands at 2860, 2917, and $2936 \mathrm{~cm}^{-1}$ in PCL spectrum are attributed to $\mathrm{C}-\mathrm{H}$ groups stretching vibration modes. Carbonyl stretching is observed at 1718 $\mathrm{cm}^{-1}$. C-O-C and C-C stretching in crystalline phase band are present at $1292 \mathrm{~cm}^{-1}$, while the same absorptions in amorphous phase are observed at $1157 \mathrm{~cm}^{-1}$. The others bands are $1238 \mathrm{~cm}^{-1}$ (asymmetric COC stretching), and 1394, and $729 \mathrm{~cm}^{-1}\left(\mathrm{CH}_{2}\right.$ rocking) (Mangiacapra et al, 2007; Elzein et al, 2004; Peng et al, 2010). The presence of LDH in PCL the nanocomposites is confirmed by the presence of additional bands bellow $800 \mathrm{~cm}^{-1}$ which are due to $\mathrm{M}-\mathrm{O}$ vibrations, Figs. $4 \mathrm{a}$ and $4 \mathrm{~b}$. Bands related to $\mathrm{O}-\mathrm{H}$ groups were observed at $3100-$ $3600 \mathrm{~cm}^{-1}$, not shown here. 
(a)

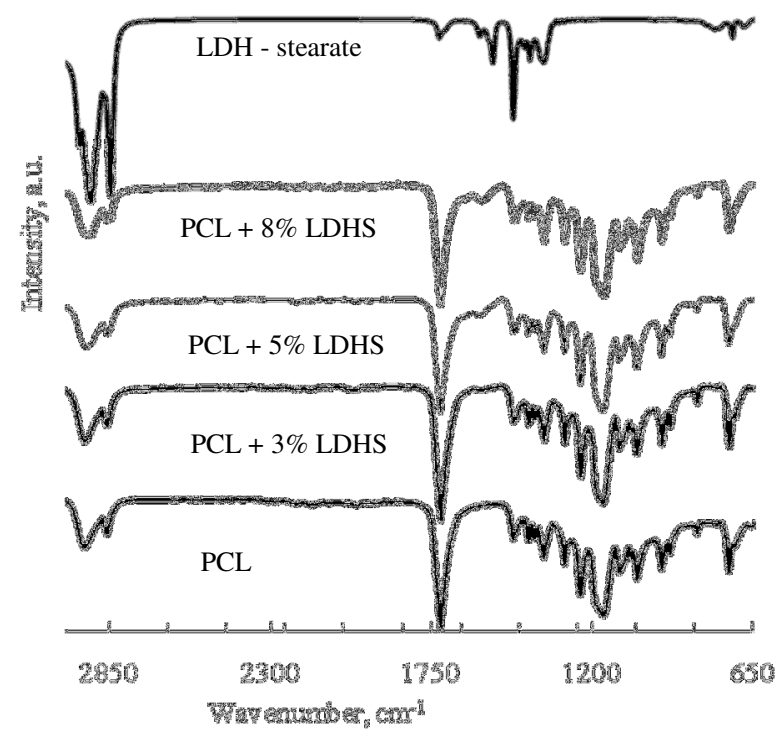

(b)

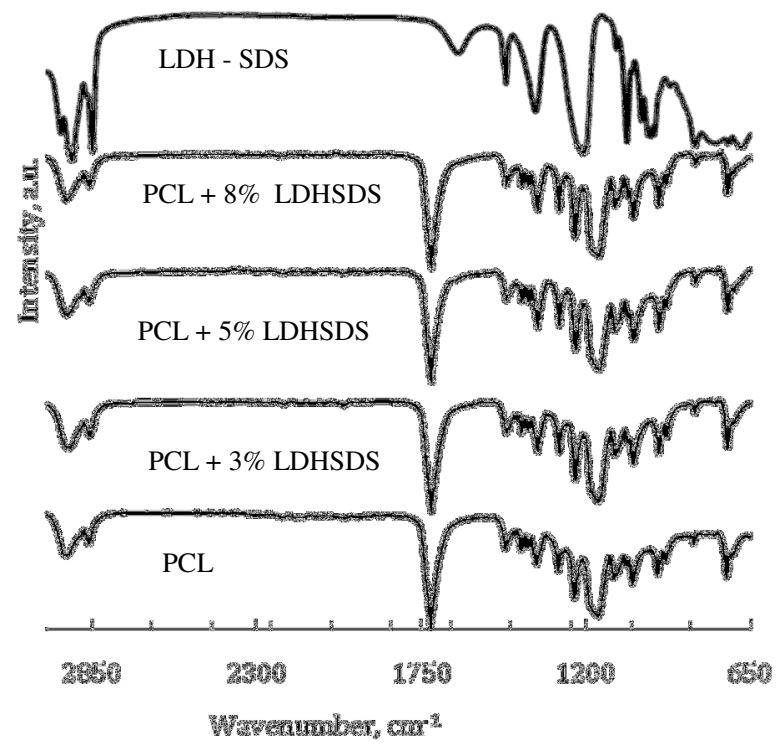

Figure 5: FTIR spectra of prepared nanocomposites. (a) Poli( $(\varepsilon)$ caprolactone - LDH stearate; (b) Poli( $\varepsilon$ )caprolactone - LDHSDS.

\subsection{Thermal properties}

Fig. 6 illustrates thermogravimetric analyses results for neat polymer and nanocomposites. Thermal degradation temperature of $\mathrm{PCL}$ composites is lower compared to the neat polymer. One of the explanations for such behaviour could be the degradation promoted by $\mathrm{OH}$ groups present in LDH structure. This was also observed Du et al (2006) in poly(propylene carbonate). Peng et al (2010) attributed such behaviour to the earlier decomposition of LDH comparated to the parent polymer. This was also observed by Nhlapo et al (2008). DTG curves (Fig. 6C and 6d) show that PCL degradation occurs in multiple steps. 

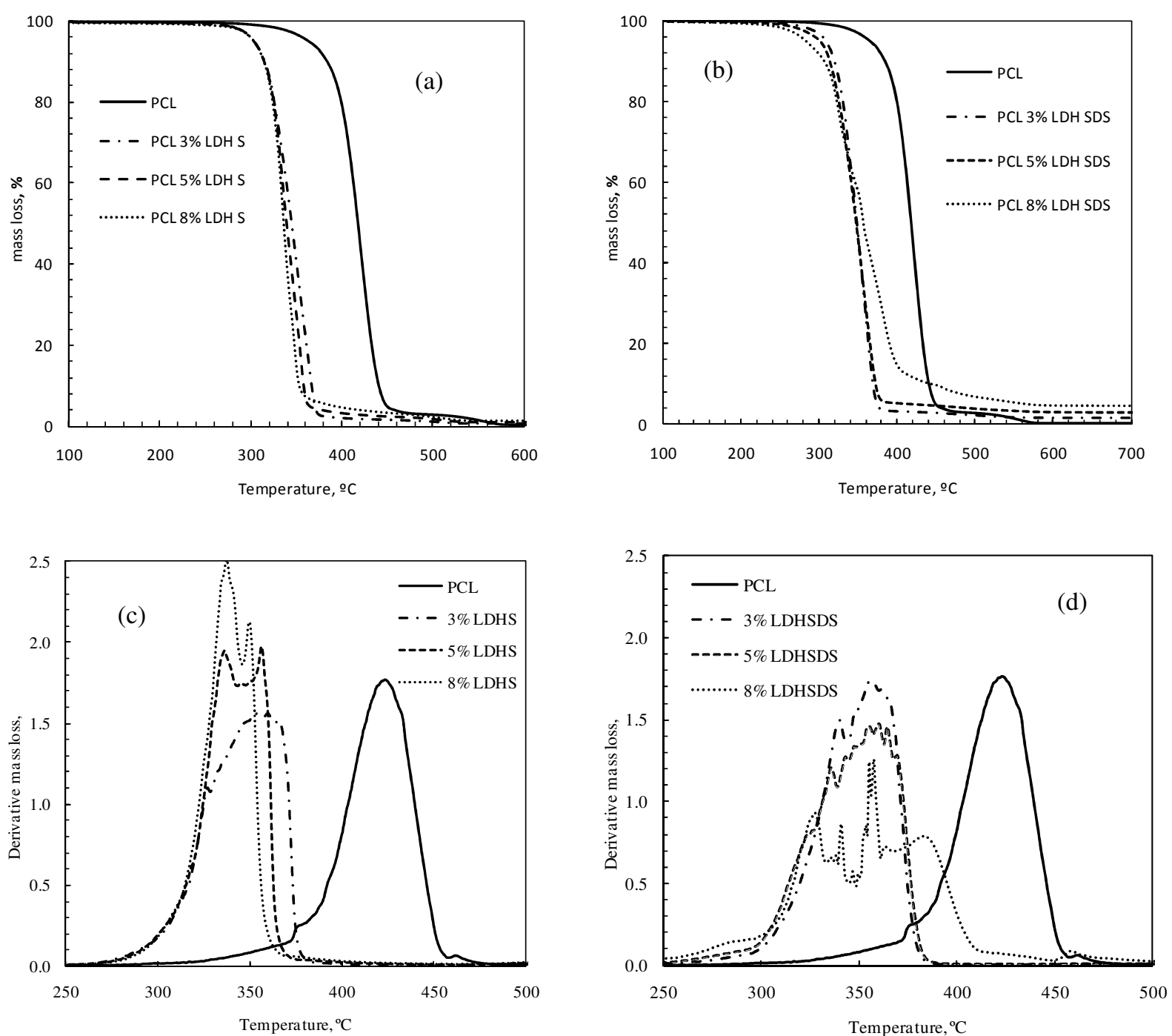

Figure 6: Temperature profile of Poli( $(\varepsilon)$ caprolactone. TG curves of PCL - LDH stearate (a), PCL - LDH SDS (b) and DTG curves of PCL - LDH stearate (c) and PCL - LDH SDS (d) nanocomposites.

Fig. 7 shows DSC curves of PCL nanocomposites and its composites prepared in the present work. Melting temperatures of both LDH - stearate and LDH - SDS nanocomposites are closer to the melting temperature of parent polymer, Fig. $7 a$ and $7 b$, suggesting that the crystal structure of PCL is maintained, as was observed by previous authors (Di et al 2003; Marras et al 2008). Crystallization behaviour, showed in Fig. 7c and 7d, indicated a slight decrease in LDH stearate nanocomposites in crystallization temperature. LDH SDS nanocomposites showed a slight increase in crystallization temperature as was expected, 29 ${ }^{\circ} \mathrm{C}$ for $3 \%$ clay loading. Above this point the crystallization temperature decreased. This was 
expected since LDH layers acts as nucleating agents as was observed for other clays. The behaviour for LDH higher loads can be understood by the explanation offered by $\mathrm{Di}$ et al (2003). The presence of organic surfactant in LDH galleries leads to interactions with PCL molecules. This effect increases with increasing LDH Concentration (Di et al 2003).
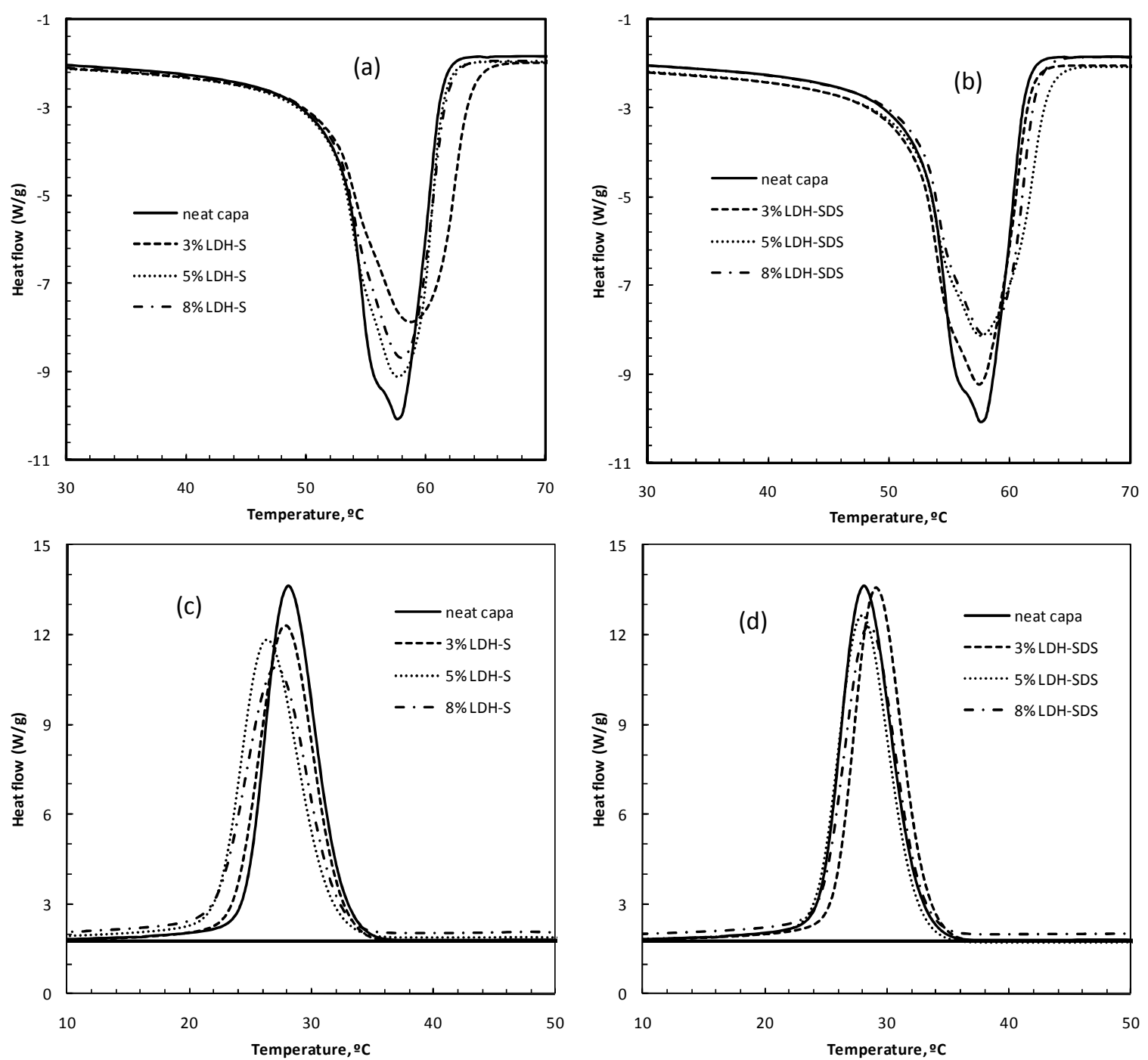

Figure 7: $\mathrm{DSC}$ curves of $\mathrm{PCL}$ and $\mathrm{PCL}$ nanocomposites. Melting endotherms of PCL-LDH stearate (a); and PCL - LDH SDS (b) nanocomposites; and crystallization exotherms of PCLLDH stearate (c); and PCL - LDH SDS (d) nanocomposites.

\section{Conclusions}

PCL LDH stearate and LDH SDS nanocomposites were prepared via melt intercalation. Nanocomposites morphology was studied by XRD and TEM while thermal properties were studied by TG and DSC. FTIR studies indicated the presence of LDH in PCL matrix. XRD and 
TEM results indicated a mixed morphology. Both systems showed the existence of a dispersed (exfoliated) LDH platelets as well as of tactoids. A good matching polymer-filler properties was observed in LDH stearate nanocomposites. TEM results indicated a better dispersion of LDH in this latter system. DSC results showed improvement in thermal properties in nanocomposites with LDH loadings up to $5 \% .8 \%$ clay loadings presented poor thermal properties compared to neat PCL.

\section{Acknowledgements}

This work is based on research supported by the National Research Foundation (NRF) through the Institutional Research Development Programme (IRDP); the South Africa/Mozambique Collaboration Programme, and the South Africa/Germany Research Collaboration Programme. Financial support from the Bundesministerium für Forschung (BMF) and the Leibniz Institute for Polymer Research, Dresden is also gratefully acknowledged.

\section{References}

Chaudhary, D.S., Prasad, R., Gupta, R.K., and Bhattacharya, S.N., 2005. Clay intercalation and influence on crystallinity of EVA-based clay composites. Thermochimica acta, 433:187-195.

Clearfield, A., et al, 1991. Intercalation of Dodecyl Sulphate into Layered Double Hydroxides. Journal of Inclusion Phenomena and Molecular Recognition in Chemistry, 11:316-378.

Costa, F.R., Abdel-Goad, M., Wagenknecht, U., and Heinrich, G., 2005. Nanocomposites based on polyethylene and Mg-Al layered double hydroxide. I. Synthesis and characterization. Polymer, 46:4447-4453.

Di, Y., lannace, S., Di Maio, E., and Nicolais, L., 2003. Nanocomposites by melt intercalation based on polycaprolactone and organoclay. Journal of Polymer Science: Part B: Polymer Physics, 41:670-678.

Du, L., Qu, B., Meng, Y., and Zhu, Q., 2006. Structural characterization and thermal and mechanical properties of poly(propylene carbonate)/MgAl-LDH exfoliation 
nanocomposite via solution intercalation. Composites Science and Technology, 66:913-918.

Elzein, T., Nasser-Eddine, M., Delaite, C., Bistac, S., and Dumas, P., 2004. FTIR study of polycaprolactone chain organization at interfaces. Journal of Colloid and Interface Science, 273:381-387.

Mangiacapra, P., Raimondo, M., Tammaro, L., Vittoria, V., Malincolo, M., and Laurienzo, P., 2007. Nanometric dispersion of a Mg/Al layered double hydroxide into a chemically modified polycaprolactone. Biomacromolecules, 8:773-779.

Marras, S.I., Kladi, K.P., Tsivintzelis, I., Zuburtikudis, I., and Panayiotou, C., 2008. Biodegradable polymer nanocomposites: The role of nanoclays on the thermomechanical characteristics and the electrospun fibrous structure. Acta Biomaterialia, 4:756-765.

Nhlapo, N., Motumi, T., Landman, E., Verryn, S., and Focke, W.W., 2008. Surfactant-Assisted Fact Acid Intercalation of Layered Double Hydroxides. Journal of Materials Science, 43:1033-1043.

Krishnamoorti, R., and Silva, S., 2000. Rheological Properties of Polymer-Layered Silicate Nanocomposites. In Polymer-Clay Nanocomposites T.J. Pinnavaia G.W. Beall Edition. John Wiley and Sons Ltd., pp 315-343.

Pavlidou, S., and Papaspyrides, 2008. A review on polymer-layered silicate nanocomposites. Progress in Polymer Science, 33:1119-1198.

Peng, H., Han, Y., Liu, T., Tjiu, W.C., and He, C., 2010. Morphology and thermal degradation behaviour of highly exfoliated CoAl-layered double hydroxide/polycaprolactone nanocomposites prepared by simple solution intercalation. Thermochimica Acta, $502: 1-7$

Wang, Y., Rodriguez-Perez, M.A., Reis, R.L., and Mano, J.F., 2005. Thermal and Thermomechanical Behaviour of Polycaprolactone and Starch/Polycaprolactone Blends for Biomedical Applications. Macromolecular Materials and Engineering, 290:729-801.

Wang, X., Rathore, R., Songtipya, P., Jimenez-Gasco, M.M., Manias, E., and Wilkie, C.A., 2011. EVA-layered double hydroxide (nano)composites: Mechanism of fire retardancy. Polymer Degradation and Stability, 96:301-313. 
Woodruff, M.A., and Hutmacher, D.W., 2010. The return of a forgotten polymer Polycaprolactone in the $21^{\text {st }}$ century. Progress in Polymer Science, 35:1217-1256.

Vaia, R.A., Ishii, H., and Giannelis, E.P., 1993. Synthesis and Properties of Two-Dimensional Nanostructures by Direct Intercalation of Polymer Melts in Layered Silicates. Chemistry of Materials, 5:1694-1696.

Vaia, R.A., and Giannelis, E.P., 1997. Lattice Model of Polymer Melt Intercalation in Organically Modified Layered Silicates. Macromolecules, 30:7990-7999.

Zanneti, M., Camino, G., Thomann, R., and Mülhaupt, R., 2001. Synthesis and thermal behaviour of layered silicate-EVA nanocomposites. Polymer, 42:4501-4507. 